\title{
Tonal expectations influence pitch perception
}

\author{
F. Marmel and B. Tillmann \\ Université de Lyon, CNRS UMR 5020, Lyon, France \\ AND \\ W. J. Dowling \\ University of Texas, Dallas, Texas
}

\begin{abstract}
In this study, we investigated the influence of tonal relatedness on pitch perception in melodies. Tonal expectations for target tones were manipulated in melodic contexts while controlling sensory expectations, thus allowing us to assess specifically the influence of tonal expectations on pitch perception. Three experiments provided converging evidence that tonal relatedness modulates pitch perception in nonmusician listeners. Experiment 1 showed, with a rating task, the influence of the tonal relatedness of a target tone on listeners' judgments of tuning/mistuning. Experiment 2 showed, with a priming task, that pitch processing of in-tune tones was faster for tonally related targets than for less related targets. Experiment 3 showed, with a comparison task, that discrimination performance for small mistunings was better when the to-be-compared tones were tonally related to the melodic context. Findings are discussed in relation to psychoacoustic research on contextual pitch perception and to studies showing facilitation of early processing steps via knowledge- and attention-related processes.
\end{abstract}

Listening to music involves not just hearing successive sounds, but also expecting future events on the basis of previous events. Western tonal music contains complex regularities that elicit expectations about future musical events. Musical regularities are based both on simple patterns, such as tone repetition, melodic contour, and interval size, and on more abstract patterns, such as tonal structure. The former elicit low-level, bottom-up expectations (referred to hereafter as sensory expectations), and the latter elicit cognitive, knowledge-based expectations (referred to hereafter as $\operatorname{cog}$ nitive expectations). Numerous studies have highlighted the role of tonal structure on musical processing (for reviews, see Bigand \& Poulin-Charronnat, 2006; Francès, 1958/1988; Krumhansl, 1990; Tillmann, Bharucha, \& Bigand, 2000). One of the key findings has been that even nonmusicians have acquired implicit knowledge of the abstract regularities defining tonal structure and that this knowledge allows for cognitive tonal expectancy formation. However, it remains to be shown which levels of music processing are influenced by cognitive expectations and whether these expectations can have perceptual effects. In the present study, we investigated the influence of listeners' tonal expectations on pitch processing. Using musical materials whose perception invoked listeners' tonal knowledge allowed us to investigate the influence of higher level, top-down processes on lowlevel perceptual processes. Studying the perceptual effects of tonal expectations thus extends psychoacoustic research that investigates - in addition to the operation of peripheral processors - the influence of more central factors on sound processing (e.g., Watson \& Foyle, 1985).
Cognitive expectations in music perception can be linked to the tonal structure of the Western musical system. Tonal structure refers to an abstract system of relations among musical tones, which can be described in terms of contextually determined probabilities of occurrence (including association strengths). In the Western tonal system, 12 pitch classes $(C, C \sharp / D$, D, D $\# / E b, E, F, F \sharp / G b, G, G \sharp / A b, A$, $A \sharp / B b$, and $B$ ) are organized into subsets of 7 pitch classes that define tonalities (or keys). Inside a key, the 7 pitch classes are hierarchically organized in accordance with the importance of their tonal function. The highest rank in the tonal hierarchy is the tonic (i.e., the first degree of the musical scale), which is the most stable tonal function and represents the anchor point of a key (Krumhansl, 1990). The tonic usually serves as the terminal event in a musical phrase and produces a sense of closure and completion. In the tonal hierarchy, the tonic is followed by the dominant (the fifth degree of the scale), the mediant (the third degree), the subdominant (the fourth degree) and then the other in-key tones, with the leading tone (the seventh degree) being at the lowest rank in the tonal hierarchy among the in-key tones. Since the tonal system is based on a restricted set of 12 tones, the structural role of a given tone depends on the tonal hierarchy instilled by a given key context. The same tone occurs in several keys, and its tonal role (i.e., its tonal function) changes depending on the other tones of the context and its underlying key. For example, the tone $\mathrm{C}$ functions as the tonic in the key of $\mathrm{C}$ major (when the subset of tones included in the scale is $\mathrm{C}$, D, E, F, G, A, B), but as the subdominant (fourth degree)

F. Marmel, frederic.marmel@olfac.univ-lyon1.fr 
in the key of G major (used subset: G, A, B, C, D, E, F \#). In what follows, a tone that has a high rank in the tonal hierarchy of the context key (e.g., the tone $\mathrm{C}$ in $\mathrm{C}$ major) is referred to as related to the tonal context, and a tone with a lower rank (e.g., the tone $\mathrm{C}$ in $\mathrm{G}$ major) is referred to as less related to the tonal context.

\section{Tonal Expectations}

In musical pieces, the tonal hierarchy is reflected in the statistical regularities of tone use: Tones belonging to a key occur more frequently together than out-of-key tones, and those with central tonal functions (e.g., the tonic and dominant) are used more often than the less central ones (Francès, 1958/1988; Krumhansl, 1990). The tonal hierarchy is thus correlated with the tones' probabilities of occurrence, and it has been hypothesized that listeners acquire implicit knowledge of tonal structure by internalizing the frequency of co-occurrence and occurrence of tonal events. Consequently, sensory information in a musical context (i.e., which tones occur more frequently in the context) is entwined with tonal structure. The investigation of listeners' implicit knowledge and of musical expectations linked to tonal hierarchyl thus gives rise to a methodological difficulty and requires the control of the sensory information in the material. In addition to sensory information linked to frequencies of occurrence (i.e., tone repetition), other features (namely, intervals and melodic contour), which do not involve the tonal hierarchy, influence listeners' expectations. These features elicit expectations according to Gestalt principles: A melodic interval generates strong expectations for successive tones to lie close in pitch height or to change contour direction (i.e., principles of pitch proximity and pitch reversal; Narmour, 1990; Schellenberg, Adachi, Purdy, \& McKinnon, 2002). These gestalt-based expectations are likely to influence tonal expectations and, thus, have to be controlled in experimental material used to investigate cognitive expectations.

In focusing on cognitive expectations (i.e., top-down expectations linked to implicit knowledge of the tonal hierarchy), previous research has addressed the influence of sensory components with post hoc analyses, such as regression analyses (Bigand, 1997; Bigand \& Pineau, 1997; Frankland \& Cohen, 1990; Hébert, Peretz, \& Gagnon, 1995), or has controlled for sensory features directly in the experimental material (e.g., Bigand, 1997; Krumhansl, 1979). The findings reveal that - beyond sensory factors such as tone repetition and pitch proximity - listeners' knowledge of the tonal hierarchy influences melody perception. For example, when two melodies differ only in their initial few tones, musical tension judgments reveal that the remaining tones (which are the same in the two melodies) are perceived differently and according to their tonal functions in the key invoked at the beginning (Bigand, 1997).

Cognitive expectancies and their influence on processing speed have been investigated with the musical-priming paradigm. This paradigm manipulates the tonal relatedness between a prime context (i.e., a chord or a chord sequence) and a to-be-processed target chord and compares tonally related targets with less related targets. Related targets should be more expected in the priming context and, thus, should be processed more quickly than less related targets. To focus on cognitive priming, the experimental material controls for acoustical overlap between the prime and the target. After single-chord primes, related targets are processed more quickly than unrelated targets, even when the primes and the targets do not share component tones (Bharucha \& Stoeckig, 1987) or are separated by a white noise burst (Tekman \& Bharucha, 1992). After seven-chord sequences, the processing of related targets is facilitated even when less related targets share more tones with the priming context (Bigand, Poulin, Tillmann, Madurell, \& D'Adamo, 2003) or when they are immediately preceded by an identical chord (Bigand, Tillmann, Poulin-Charronnat, \& Manderlier, 2005).

In sum, tonal expectations influence musical event processing - notably, with shorter response times for tonally related, more expected musical events than for less related, less expected ones. The aim of our study was to investigate the levels of music processing influenced by tonal expectations and to focus on the accuracy of pitch perception.

\section{Contextual Pitch Perception}

Psychoacoustic research on pitch perception has investigated the influence of listeners' expectations in nonmusical contexts. These expectations have been based mostly on features of the experimental session, such as the probability of occurrence of a specific pitch (Tanner \& Norman, 1954) or the use of cues indicating the pitch of the to-be-detected signal (Greenberg \& Larkin, 1968; Hafter, Schlauch, \& Tang, 1993; Howard, O'Toole, Parasuraman, \& Bennett, 1984; Watson \& Foyle, 1985; Watson, Kelly, \& Wroton, 1976). In Greenberg and Larkin, listeners' expectations were manipulated by presenting a cue tone whose frequency corresponded to the frequency of the to-be-detected signal on $70 \%-80 \%$ of the trials. Results of this probe signal method revealed best detection performance for the signal at the cued (expected) frequency, and performance decreased for unexpected frequencies as a function of the distance from the cue tone. Facilitated pitch processing has been observed also with indirect cues that consisted of either one tone (Hafter et al., 1993) or a tone sequence (Howard et al., 1984). In Hafter et al., pitch processing was facilitated by cues that were a musical fifth (a 3/2 frequency ratio) away from the targets, showing that the cue need not rely on direct sensory priming of the target pitch. For longer tone contexts, pitch processing was facilitated when participants could develop expectations for target pitches on the basis of the pattern structure in the context (Howard et al., 1984). For example, in continuously rising (or falling) 12-tone sequences, the 11th tone (the to-be-detected target) was expected (or not) in terms of the homogeneous intervallic succession in the sequence, and detection performance was better for expected targets (i.e., tones in the continuity of the pattern) than for unexpected targets violating the pattern (Howard et al., 1984).

Pitch processing has also been investigated in musical contexts. Comparing native and nonnative musical contexts, Lynch and Eilers (1992) reported that Western nonmusicians detected mistuned notes more accurately with melodies based on a Western tonal scale than with those 
based on an unfamiliar scale (in this case, a Javanese pelog scale). Facilitated pitch processing by Western listeners in Western tonal scale contexts (vs. non-Western ones) has also been reflected in electrophysiological data showing larger amplitudes of mismatch negativity (MMN; Brattico, Näätänen, \& Tervaniemi, 2001). Focusing on Western tonal contexts, Francès (1958/1988, Experiment 2) asked musician listeners to detect mistuned tones that were anchored to lower or higher tones. Results showed that mistunings were detected better when the direction of mistuning conflicted with the expected direction of tone motion than when it was coherent with it.

When pitch perception is rendered more difficult due to timbral changes of the involved tones, a tonal context can increase pitch perception accuracy. In Warrier and Zatorre (2002, Experiment 1), pairs of tones that differed in pitch (by 17, 35, or 52 cents [a cent is 1/100 of a semitone in logarithmic units]) and/or timbre (more or less bright) were presented in isolation or preceded by a melodic context in which the targets functioned as the tonics. Listeners rated the degree of difference between the tones (for the pairs) or the degree of mistuning (for the melodies). Melodic contexts improved pitch extraction, in comparison with single tone contexts (see also Brattico et al., 2001). Comparisons with a nonmelodic context (with the same tone repeated five times) and with random, atonal contexts revealed that the facilitation in tonal contexts was due not only to the presence of other tones or to higher structural complexity, but also to the tonal structure of the context (Warrier, Belin, Merlet, \& Zatorre, 1999; Warrier $\&$ Zatorre, 2002). These findings suggest that the tonal context creates tonal reference points allowing for more efficient pitch extraction in spite of the timbral changes.

In our study, instead of comparing melodic contexts to no context or to a random context, we investigated the influence of degrees of tonal relatedness on pitch processing by using only tonal melodies that were similar in structure complexity. More specifically, our aim was to investigate the influence of listeners' tonal expectations on pitch per- ception for target tones differing in tonal function. The target (i.e., the last tone of the melodies) functioned either as the tonic, which is the cognitive reference point of a tonality (Krumhansl, 1990), or as the subdominant, which is less related but still important in the tonal hierarchy. The melodic contexts were constructed in pairs to control for sensory differences (such as tone repetition and melodic contour) and to focus on the cognitive components of tonal expectations and their influence on pitch perception. In the related condition with the expected tonic target, pitch perception should be facilitated, in comparison with the less related condition with the less stable, less expected subdominant target. In Experiment 1, we used ratings of degree of mistuning (proposed by Warrier \& Zatorre, 2002). In Experiment 2, we used a speeded reaction time task requiring binary judgments of mistuning. In Experiment 3 , we asked participants to make same/different judgments on tone pairs that defined the end of the melodies (with one tone being mistuned or not). In contrast to psychoacoustic studies, expectations for the target pitch depended on the key invoked by the melodic context and required listeners' tonal knowledge.

\section{EXPERIMENT 1}

\section{Method}

Participants. Twenty students from the University of Lyon participated in Experiment 1. Musical practice, as measured by years of instrumental instruction, varied from 0 to 11 years, with a mean of 4.0 years $(S D=4.1)$ and a median of 3.0.

Materials. Twelve pairs of melodies like the example shown in Figure 1 were composed. All the melodies had a length of two bars of four beats each. The two melodies of a pair had exactly the same rhythmic patterns. The 12 melodic pairs had the same global rhythmic pattern: The first three beats of each bar consisted of 8th and/or 16 th notes, the fourth beat was a quarter note, and the same rhythmic pattern was used for the two bars of each melody. The precise rhythmic figures used in a bar are shown in Figure 2. This diversity in rhythms for the three first beats was introduced to prevent the set of melodies from being too repetitive and boring for the participants. The different rhythmic figures resulted in slight variations in the number of tones in a melody $(M=15.2, S D=2.1)$. The two melo-

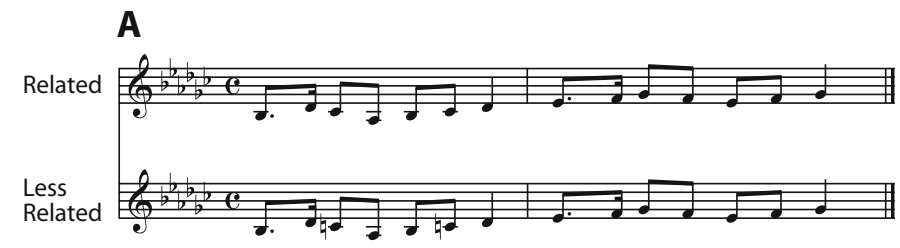

B

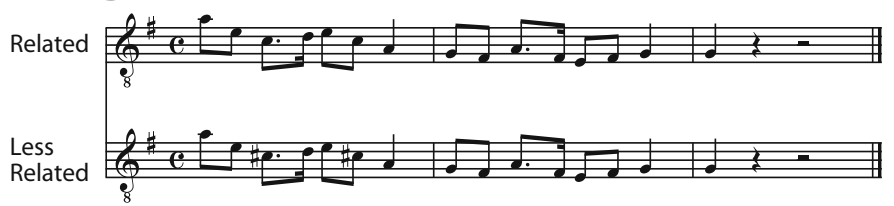

Figure 1. (A) Example of the 12 pairs of melodies used in Experiments 1 and 2. (B) The same melodies were used in Experiment 3, but with the last tone repeated. The tone differing between related and less related melodies can be visually identified by the alteration marks. Examples of sound materials are available at olfac.univ-lyon1.fr/bt-sound.html. 
dies of a pair, in addition to having exactly the same rhythms, had almost the same tones. The two melodies of a pair differed only by one, possibly repeated, tone in the first bar. The serial position of this changed note was varied across melodic pairs from the second 8th note of the first beat to the fourth beat. This note change modified the tonal function of the target (the last tone of the melody, which was always a quarter note) to be either the tonic (related target) or the subdominant (less related target). Twelve melodic pairs were composed so that each of the 12 major keys was represented twice: once with a melody ending on a tonic and once with a melody ending on a subdominant. Five additional melodies were used as examples during the task instructions.

Apparatus. The melodies were created in MIDI with Cubase SX2 software (Steinberg) and were transformed into audio files using The Grand (a VST piano instrument by Steinberg). MIDI velocity was constant for all pitches. The melodies were recorded at a tempo of $789.5 \mathrm{msec}$ per beat without any expressive or stylistic timing variations. This tempo represents a duration of $789.5 \mathrm{msec}$ for a quarter note, $394 \mathrm{msec}$ for an 8th note, and $197 \mathrm{msec}$ for a 16th note. The overall duration of a melody was 7,000 msec (including the fadeout for the final target tone that increased its length to $1,474 \mathrm{msec}$ ). Cubase's microtuner was used to create the melodies with the last tone either in tune or shifted in pitch by \pm 17 or \pm 35 cents. These two degrees of mistuning were selected from Warrier and Zatorre (2002) and represent a $\pm 1 \%$ or $2 \%$ deviation in frequency. (A semitone represents a $5.946 \%$ frequency difference.) The experiment was run on PsyScope software (J. [D.] Cohen, MacWhinney, Flatt, \& Provost, 1993).

Procedure. The experiment consisted of a training phase and an experimental phase. In the training phase, the concept of out-of-tune notes was explained to the participants with a familiar French song ("J'ai du bon tabac") that was played first with its last tone in tune and then with its last tone shifted in pitch by -35 cents, +35 cents, -17 cents, and +17 cents. The 35 -cent pitch shifts were presented as very out of tune, and the 17-cent pitch shifts as slightly out of tune. The participants were familiarized with the task with the five sample melodies. The participants had to indicate their response by pressing a key on the computer keyboard. If they judged the target tone to be in tune, they pressed the in-tune key ("bonne"). If they judged it to be out of tune, they had to grade their judgment on a 3-point scale: slightly out of tune ("légèrement fausse"), out of tune ("fausse"), or very out of tune ("très fausse"). In the experimental phase, the participants judged the target tones of 120 melodies. The 12 pairs of melodies, which consisted of 12 related and 12 less related melodies, were presented with the last note in tune, 17 cents lower or higher, and 35 cents lower or higher $(12 * 2 * 5=120)$. The melodies were presented in a different random order for each participant. The participants had $3 \mathrm{sec}$ to answer, and then a $250-\mathrm{msec}$ noise mask was presented. No feedback was given. The participants pressed another key to proceed to the next trial.

Data analysis. The participants' ratings were coded on a $0-3$ scale, with 0 corresponding to in-tune judgments, 1 to slightly out of tune, 2 to out of tune, and 3 to very out of tune. The results were first analyzed with mean ratings averaged over the sequence set for each condition and participant (as in Warrier \& Zatorre, 2002). We then analyzed discrimination performance between in-tune tones and each of the four mistuned conditions with areas under the receiveroperating characteristic (ROC). The area under the ROC provides an unbiased estimate of discrimination performance, where chance is .50 (Swets, 1973), and has the advantage of preserving more response information than does $d^{\prime}$ or $A^{\prime}$ and of being uncorrelated with measures of response bias (unlike $d^{\prime}$; see Dowling, Kwak, \& Andrews, 1995; Verde, Macmillan, \& Rotello, 2006). In addition, to assist in interpreting area scores, we analyzed hits and false alarms (with hit and false alarm rates being percentages of in-tune responses to the five types of test stimuli).

Each of the mean ratings and hit and false alarm rates was analyzed with a $2 \times 5$ ANOVA, with tonal relatedness (tonic or subdominant) and pitch deviation $(0,+17$ cents, -17 cents, +35 cents, or

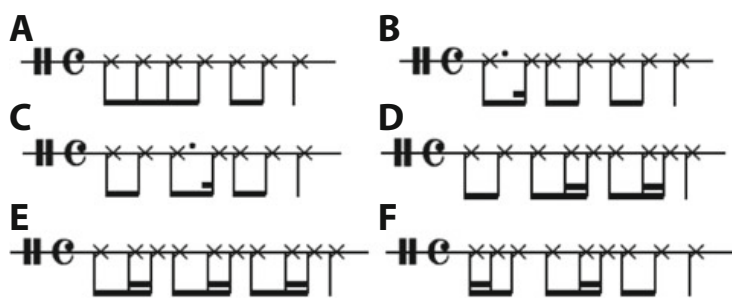

Figure 2. Rhythmic figures used in the melodic set. For all melodies, the second bar had exactly the same rhythm as the first bar, so only one bar is displayed. Of the 12 melodic pairs, 3 had the rhythmic figure shown in panel $\mathrm{A}, 4$ had the rhythmic figure in $\mathrm{B}$, 2 had the rhythmic figure in $C, 1$ had the rhythmic figure in $D, 1$ had the rhythmic figure in $\mathrm{E}$, and 1 had the rhythmic figure in $\mathrm{F}$.

-35 cents) as within-participants factors. Area scores were analyzed with a $2 \times 4$ ANOVA, with tonal relatedness (tonic or subdominant) and pitch discrimination $(0 /+17,0 /-17,0 /+35$, or $0 /-35$ cents $)$ as within-participants factors. The application of the Greenhouse-Geisser correction in these analyses did not change the significance patterns.

\section{Results}

For mean ratings (Figure 3 ), the main effect of tonal relatedness was significant $\left[F(1,19)=6.41, M S_{\mathrm{e}}=.044\right.$, $p<.05]$. Mean ratings were higher for less related targets than for related targets, indicating that target tones were judged as being more out of tune when they functioned as subdominants than when they functioned as tonics. The main effect of pitch deviation was significant $[F(4,76)=$ $\left.111.24, M S_{\mathrm{e}}=.20, p<.0001\right]$. Deviations of -35 cents were judged more out of tune than were deviations of +35 cents, followed by deviations of -17 cents and deviations of +17 cents, and in-tune tones received the lowest values. This hierarchy in ratings was significant by post hoc Tukey HSDs $(p s<.01)$. The interaction be-

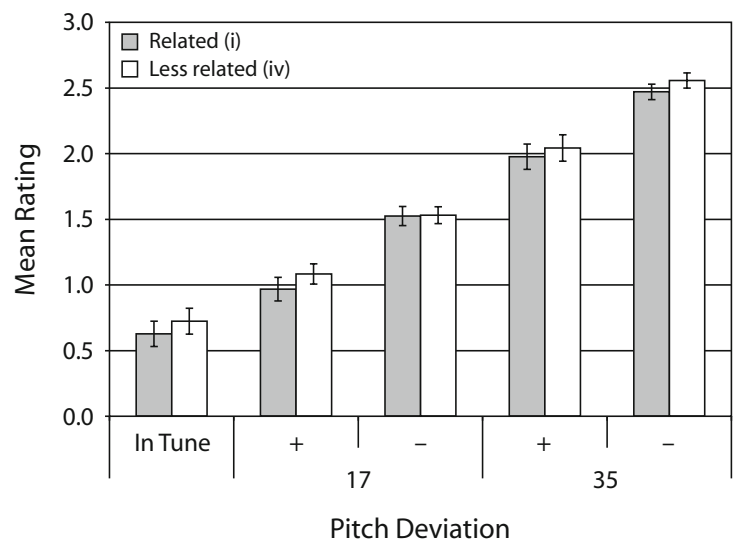

Figure 3. Mean ratings of Experiment 1 presented as a function of tonal relatedness (related tonic, $i$; less related subdominant, iv) and pitch deviation (no pitch deviation $=$ in tune, degrees of pitch deviation $\pm 17, \pm 35$ cents). On the rating scale, 0 represents in-tune responses and 1 to 3 represent out-of-tune responses (from 1 , slightly out of tune to 3, very out of tune). Error bars represent standard errors. 
tween tonal relatedness and pitch deviation was not significant $(F<1)$.

For area scores (Figure 4$)$, the main effect of tonal relatedness was significant $\left[F(1,19)=5.05, M S_{\mathrm{e}}=.015\right.$, $p<.05]$. Performance was better for related targets than for less related targets, indicating that in-tune targets were more accurately discriminated from out-of-tune targets when they functioned as tonics than when they functioned as subdominants. The main effect of pitch discrimination was significant $\left[F(4,57)=78.0, M S_{\mathrm{e}}=.0074, p<.0001\right]$. Discrimination was better for $0 /-35$ than for $0 / 35$, followed by $0 /-17$ and $0 / 17$ cents. This hierarchy in ratings was significant by post hoc Tukey HSDs $(p s<.01)$. The interaction between tonal relatedness and pitch deviation was not significant $(p=.10)$.

For hits and false alarms (Figure 5), the main effect of tonal relatedness was significant $\left[F(1,19)=5.60, M S_{\mathrm{e}}=\right.$ $.009, p<.05]$, indicating that the participants answered in tune more often when the targets were related tonics. The main effect of pitch deviation was significant $\left[F(4,76)=55.3, M S_{\mathrm{e}}=.035, p<.0001\right]$. Post hoc Tukey HSDs showed significantly higher percentages of in-tune responses for in-tune targets than for +17 -cent targets and for +17 -cent targets than for -17 -cent targets $(p \mathrm{~s}<$ $.001)$. The interaction between tonal relatedness and pitch deviation was not significant $(p=.12)$.

\section{Discussion}

In Experiment 1, we investigated the influence of tonal relatedness on listeners' judgments of tuning/mistuning of a target tone. This influence was studied with graduated pitch judgments, as previously used by Warrier and Zatorre (2002). Since the tonal function of the target tone was manipulated by changing only one (possibly repeated) tone at the beginning of the melodic context, the influence of context on listeners' responses was linked to tonal, cognitive-based expectations, and not to features such as pitch repetition, interval, or contour.

The participants' judgments showed an influence of tonal relatedness on pitch perception in terms of mean ratings, area scores, and hits/false alarms. The mean ratings and hit/false alarm analyses showed that the lower rank in tonal hierarchy of the subdominant targets increased listeners' feeling that a mistuned target was out of tune and decreased the feeling that an in-tune target was in tune (and the reverse for tonic targets). The lack of interaction between tonal relatedness and pitch deviation in mean ratings and hits/false alarms indicated the contribution of a response bias shift: The participants attributed more mistuning to less related targets and were more tolerant of pitch deviations for related targets. However, area scores, which provide an unbiased estimate of performance (Swets, 1973), indicated not only that the influence of tonal relatedness involved a shift in response bias, but also that discrimination performance between in-tune and out-of-tune targets was better for tonic targets than for subdominant targets.

The analyses thus revealed two influences in the participants' responses: increased discrimination of pitch tunings for tonics than for subdominants (as indicated by the

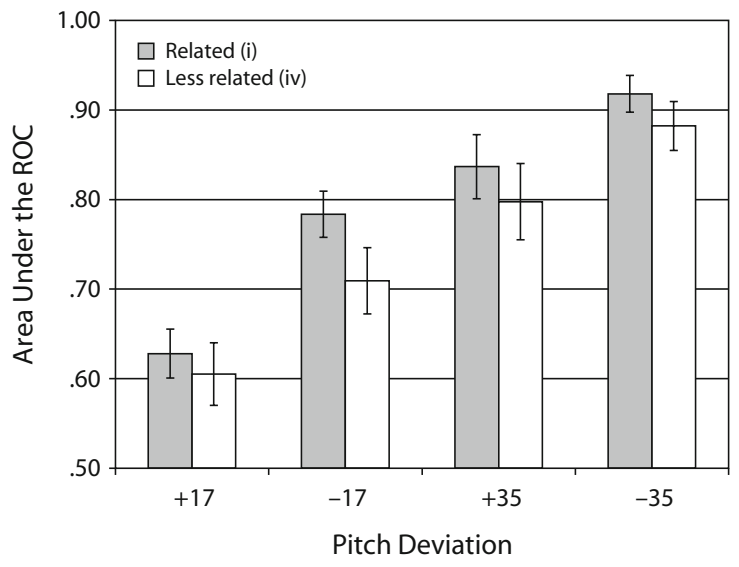

Figure 4. Areas under the receiver-operating characteristics (ROCs) for Experiment 1 presented as a function of tonal relatedness (related tonic, $i$; less related subdominant, iv) and pitch discrimination $(0 /+17,0 /-17,0 /+35,0 /-35$ cents $)$. Chance level is at $\mathbf{. 5}$. Error bars represent standard errors.

area scores) and a response bias to attribute more mistuning to subdominants than to tonics (as indicated by mean ratings and hits and false alarms). The differences in area scores suggest that a difference in tonal function affects pitch perception. The melodic context with its tonal structure (i.e., invoked key, implied tonal hierarchy) may give the tonic target the role of a cognitive reference point and may facilitate the accuracy of mistuning judgments. This finding extends the observation by Warrier and Zatorre (2002): Pitch perception not only is facilitated by a tonal context (in comparison with no context or a random tone context), but also is influenced by the tonal function that a tone takes on inside this context (notably, with a facilitation of the tonic).

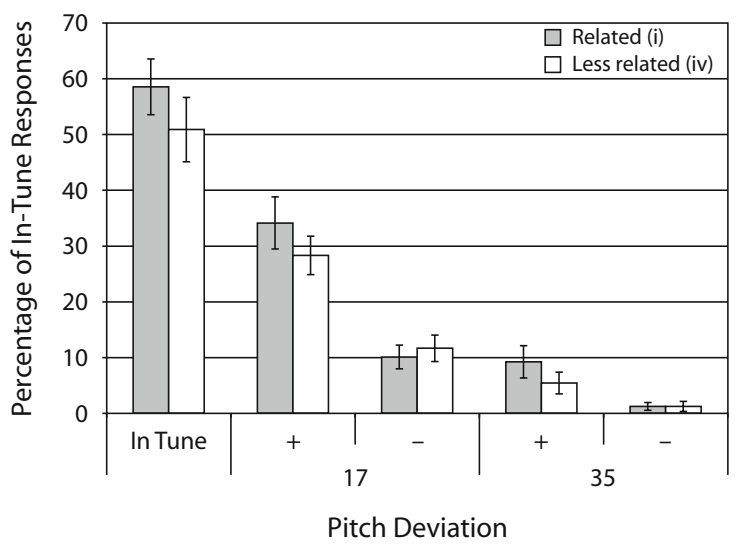

Figure 5. Hits and false alarms in Experiment 1 presented as a function of tonal relatedness (related tonic, $i$; less related subdominant, iv) and pitch deviation (in tune, $\pm 17, \pm 35$ cents). Hits are the percentages of in-tune responses in the in-tune condition. False alarms are the percentages of in-tune responses in the \pm 17 and \pm 35 -cent conditions. Error bars represent standard errors. 
Response bias might have been influenced by the instructions presenting concepts of in tune and out of tune with the French words for good and wrong (bonne and fausse), which may have been ambiguous for the musically inexperienced participants. Listeners may have confounded a wrong note in pitch and a wrong note in terms of tonal relatedness, and this then may have biased them to judge tones as more out of tune when they were less related to the tonal context. In other words, the weaker tonal function of subdominants may have given the impression of a tonal mistuning that was confused with the pitch mistuning that the listeners had to judge. A further indication of the ambiguity of the instructions may be found in the mean ratings showing that in-tune tones were still heard as somewhat out of tune (0.65-0.7 in Figure 3). Since this is observed even for in-tune tonic targets, the participants may also have used features other than tonal relatedness in making their responses. Even if the to-be-judged pitch height is an objective property of a tone, this tendency may reflect the subjective noise in the perception and evaluation of an event. The task used in Experiment 3 aimed to focus on the influence of tonal relatedness on pitch discrimination by eliminating a possible source of response bias.

Finally, all three analyses revealed that the direction of pitch deviation influenced the participants' judgments: Lowered pitches were perceived as more out of tune than were pitches raised by the same amount. This asymmetry is in agreement with the intonation used in musical performance: Pitch perception is less accurate for raised pitch intonation than for lowered pitch intonation, leading to more pronounced pitch deviations in performance in the direction of raised pitch (Geringer \& Madsen, 1984). Furthermore, a similar perceptual asymmetry has been observed in auditory nonmusical contexts. In a study by Gottselig, Brandeis, Hofer-Tinguely, Borbély, and Achermann (2004), participants listened to tone sequences consisting of eight 50 -msec-long pure tones. The tones' frequencies did not fall on a Western tonal scale, and the intervals between the tones did not correspond to Western tonal intervals. The frequency of the sixth tone of the sequences was raised or lowered by $85 \mathrm{~Hz}$, resulting in a deviant sequence. In a same/different discrimination task, the participants listened to pairs of sequences where the first one was always the standard and the second could be a standard or a deviant. Discrimination performance was better for the low deviants than for the high deviants, and the MMN amplitude was larger for low deviants than for high deviants. This finding suggests that the asymmetry observed in our study and in intonation research may have resulted from general (not specifically musical) auditory processes, as yet undocumented, to our knowledge.

\section{EXPERIMENT 2}

Experiment 1 showed that the tonal function of a target tone influences mistuning judgments. This paradigm required the participants to make explicit judgments by using a graduated response for the mistuned tones. Experiment 2 tested whether the tonal context effect on pitch judgments observed in Experiment 1 also influences pro- cessing times, with the hypothesis that processing times would be shorter for tonally related tones. Tonal relatedness effects on processing speed have been demonstrated with the priming paradigm for chords (e.g., Bharucha \& Stoeckig, 1986; Bigand \& Pineau, 1997). Participants make speeded binary judgments on a perceptual feature of the target chord that is manipulated independently of the target's tonal relatedness. For example, participants can make sensory consonance/dissonance judgments of targets that contain a mistuned tone (or not). In-tune judgments are faster for related targets than for unrelated ones. Experiment 2 adapted the binary in-tune/out-of-tune speeded identification task to investigate the influence of tonal relatedness on pitch perception in melodies. We expected pitch processing to be faster for the related tonic tone than for the less related subdominant tone.

\section{Method}

Participants. Twenty students from the University of Lyon participated in Experiment 2. Musical practice, as measured by years of instrumental instruction, varied from 0 to 10 years, with a mean of 1.8 years $(S D=3.1)$ and a median of 0 .

Materials. The melody pairs of Experiment 1 were used, with the last tone being played in tune or 35 cents higher. ${ }^{2}$ Eight additional melodies served as examples for the task.

Procedure. The experiment consisted of a training phase and an experimental phase. In the training phase, the concept of out of tune was explained to the participants, using a familiar French song ("J'ai du bon tabac") that was played with its last tone in tune, then repeated with its last tone shifted in pitch by -35 and +35 cents. The participants were trained with 8 sample melodies: They had to judge as quickly and as accurately as possible whether the last tone of each melody was in tune or out of tune by pressing one of two keys on the computer keyboard. Response times were measured from the onset of the target tones. In the experimental phase, the participants performed the same task with 48 melodies presented in pseudorandom orders. Pseudorandom orders were random orders with two constraints: The 2 melodies of a pair had to be separated by at least 4 melodies, and a given pitch deviation condition (in tune, out of tune) was not repeated more than five times in succession. The participants received error feedback. A 250-msec noise mask followed each trial, and the participants had to press a third key to proceed to the next trial.

Data analysis. Percentages of correct responses and response times for correct responses (averaged over the sequence set) were analyzed by two $2 \times 2$ ANOVAs, with tonal relatedness (tonic or subdominant) and pitch deviation (in tune or out of tune) as withinparticipants factors. One participant was dropped from the analyses because of low accuracy (correct responses inferior to 55\%). Data were screened for outlier response times (defined as response times that did not lie within four standard deviations from the mean); no outlier response times needed to be omitted.

\section{Results}

For percentages of correct responses (Table 1) only the main effect of pitch deviation was significant $\left[F(1,18)=6.60, M S_{\mathrm{e}}=116.45, p<.05\right]$. The participants responded more accurately to in-tune targets than to out-of-tune targets.

For response times for correct responses (Figure 6), the main effect of tonal relatedness was significant $[F(1,18)=$ 6.23, $\left.M S_{\mathrm{e}}=3,436.78, p<.05\right]$ and interacted with pitch deviation $\left[F(1,18)=4.70, M S_{\mathrm{e}}=1,737.10, p<.05\right]$. Response times were shorter for related targets than for less related targets, and this difference was significant only for 
Table 1

Percentages of Correct Responses (With Standard Errors)

in Experiment 2 Presented As a Function of Tonal Relatedness (Related Tonic, i; Less Related Subdominant, iv) and Pitch Deviation

\begin{tabular}{cccccc}
\hline \multirow{2}{*}{ Pitch Deviation } & \multicolumn{3}{c}{ Tonal Relatedness } \\
\cline { 2 - 3 } & \multicolumn{2}{c}{ Related (i) } & & \multicolumn{2}{c}{ Less Related (iv) } \\
\cline { 2 - 3 } \cline { 5 - 6 } & $M$ & & & $M$ & $S E$ \\
\hline In tune & 84.6 & 2.57 & & 81.1 & 2.10 \\
Out of tune & 76.3 & 3.62 & & 76.8 & 3.80 \\
\hline
\end{tabular}

in-tune targets $\left[F(1,18)=15.83, M S_{\mathrm{e}}=3,405.14, p<\right.$ $.001]$, and not for out-of-tune targets $(F<1)$. The main effect of pitch deviation was not significant.

\section{Discussion}

To investigate pitch processing in tonal contexts, Experiment 2 introduced a melodic-priming paradigm using intune/out-of-tune judgments. In contrast to the harmonicpriming studies that used a consonance/dissonance task on chords, the use of melodic sequences allowed us to study the processing of target tones' pitch (in contrast to processing of target chords' spectral color-i.e., presence/ absence of dissonance/roughness in target chords). The results indicate that tonal relatedness influenced speed of pitch processing: The pitch of an in-tune target was processed more quickly when tonally related to the melodic context (tonic) than when less related (subdominant). This finding extends the outcome of Experiment 1 to processing speed: In a tonal context, listeners' tonal expectations are very precise for the pitch of the tonic tone, leading to faster pitch processing of a related tonic, in comparison with a less related subdominant. The tonal hierarchy of the context (and its implied key) sets a cognitive reference point (the tonic; see Krumhansl, 1990) and induces expectations for this reference point. The pitch of the target can then be compared with this reference

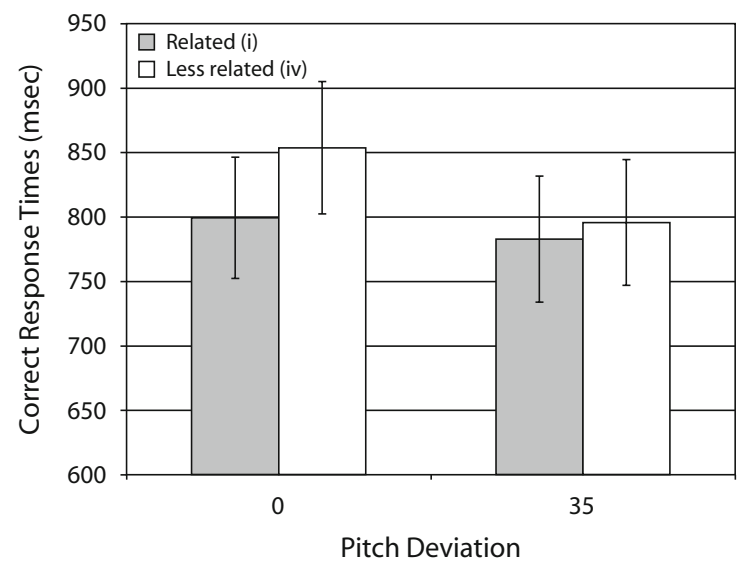

Figure 6. Correct response times in Experiment 2 presented as a function of tonal relatedness (related tonic, $i$; less related subdominant, iv) and pitch deviation (in tune, 35 cents). Error bars represent standard errors. pitch, a comparison that speeds up responses when the target matches expectations (actually is the tonic). Since the subdominant is less expected, listeners' expectations for its pitch may be less precise, and judging the correct pitch is slowed down.

Tonal relatedness interacted with the type of pitch deviation: Only in-tune tonic targets were processed significantly more quickly than subdominant targets. This difference between in-tune and out-of-tune targets is in agreement with the harmonic-priming studies in which consonance/ dissonance discrimination tasks have been used. In those studies, processing was faster for consonant-related target chords than for consonant-unrelated target chords, but this difference was smaller, absent, or even reversed for dissonant target chords (e.g., Bharucha \& Stoeckig, 1986; Bigand \& Pineau, 1997). This difference between consonant and dissonant targets has been attributed to response bias. The weaker tonal relatedness may result in a less strong integration of the target event into the tonal context (creating a contextual tonal dissonance), and this may favor the categorization of the dissonant target as dissonant, thus diminishing or counteracting the tonal-priming effect (i.e., decreasing the difference between related and less related targets). In addition, response bias may slow down in-tune judgments for less related in-tune targets, thus reinforcing the tonal priming effect (for discussions, see Bharucha \& Stoeckig, 1986; Tillmann, Bigand, Escoffier, \& Lalitte, 2006). The interaction between tonal relatedness and pitch deviation observed in Experiment 2 can be explained in a similar way: The participants may have been biased toward judging less related target tones as being out of tune because they are less well tonally integrated into the context. For less related out-of-tune targets, this bias would speed up the out-of-tune judgments, thus attenuating the tonal-priming effect. The fact that the response bias did not reverse the priming effect for out-of-tune targets (i.e., with shorter response times for less related than for related targets) may have been due to the subtle tonal relatedness manipulations, as is suggested by harmonic-priming data: For dissonant target chords, the harmonic-priming effect was less pronounced for the opposition of tonic versus subdominant chords (Bigand, Madurell, Tillmann, \& Pineau, 1999), whereas it was reversed for the opposition of tonic versus out-of-key chords (Bharucha \& Stoeckig, 1986; Tillmann, Bigand, \& Pineau, 1998).

\section{EXPERIMENT 3}

Experiments 1 and 2 showed an influence of listeners' tonal expectations on pitch judgments with the use of either a graduated scale or speeded response times. However, a potential problem with these two tasks is that listeners need to use internal reference pitches indicating the correct pitch of an in-tune tone to judge the target. This need for internal reference pitches may have enhanced possible response biases (see the Discussion sections for Experiments 1 and 2). Even though area scores in Experiment 1 revealed a bias-free influence of tonal relatedness on pitch judgments and response times in Experiment 2 cannot be solely explained by response bias, we further investigated 
the influence of tonal relatedness on pitch processing in Experiment 3 by using a same/different task. This task does not require an internal reference pitch and should also avoid the implication of tonal relatedness in the response. For this discrimination task, the last tone of the melodies (i.e., functioning as tonic or subdominant) was repeated (Figure 1B). The last tone was repeated either identically or with a slight mistuning, and the participants were asked to judge whether the last two tones were the same or different (see Johnston \& Jones, 2006, for the use of a similar paradigm in nontonal contexts). Consequently, no internal reference pitch was needed to compare the two tones. In order to calculate areas under the ROC as an unbiased estimate of listeners' pitch discrimination, Experiment 3 was designed as a rating scale signal detection theory (SDT) experiment (see Dowling, Tillmann, \& Ayers, 2001), and its aim was to assess the influence of tonal relatedness on the detection of fine mistunings.

\section{Method}

Participants. Forty-two students from the University of Lyon participated in Experiment 3. Musical practice, as measured by years of instrumental instruction, varied from 0 to 7 years, with a mean of 1.7 years $(S D=2.3)$ and a median of 0 .

Materials. The melody pairs in Experiment 1 were used, with their last tone being repeated identically or lowered in pitch by 17 or 9 cents (a $1 \%$ or $0.5 \%$ deviation in frequency, respectively). Eighteen shorter melodies, which also ended on tonic or subdominant tones, were composed for the participants in order to familiarize themselves with the task. These shorter melodies were one-and-a-half 4-beat bars long, with the last 2 beats being the repeated target tones (identical repetition or mistuned repetition). Four example melodies were adapted from the eight additional melodies in Experiment 2.

Procedure. The experiment contained a training phase and an experimental phase. In the training phase, the participants were first familiarized with the two pitch deviations by listening to tone pairs, with the tone being repeated either identically or with one of the two pitch deviations. The participants were then trained for the task with the 18 short melodies: The participants judged whether the last two tones were identical or not by using a 4-point scale $(1$, sure different; 2 , not sure different; 3 , not sure same; 4 , sure same). No time limit was imposed for responses. Finally, the participants were trained with the 4 example melodies. In the experimental phase, the participants judged 216 melodies (the 12 pairs of melodies with the target tone either in tune or lowered in pitch by 9 or 17 cents, each presented three times in three successive blocks with different pseudorandom orders; $24 * 2 * 3=216$ ). The participants received feedback on errors. Further details of the procedure were as described in Experiment 2.

Data analysis. Discrimination performance between the in-tune condition and each of the two mistuned conditions was analyzed with areas under the ROC. In addition, hits and false alarms (percentages of in-tune responses) were computed separately for each condition and participant for in-tune and out-of-tune stimuli, respectively.

\section{Results}

Area scores were analyzed by a $2 \times 2$ ANOVA, with tonal relatedness (tonic or subdominant) and pitch discrimination ( $0 / 17$ or $0 / 9$ cents) as within-participants factors. The interaction between tonal relatedness and pitch discrimination was significant $\left[F(1,41)=5.23, M S_{\mathrm{e}}=\right.$ $12.55, p<.05$; see Figure 7]. The area scores were significantly higher for tonic tones than for subdominant tones in the 0/9-cent discrimination $[F(1,41)=5.26$, $\left.M S_{\mathrm{e}}=28.89, p<.05\right]$, but not in the $0 / 17$ discrimination

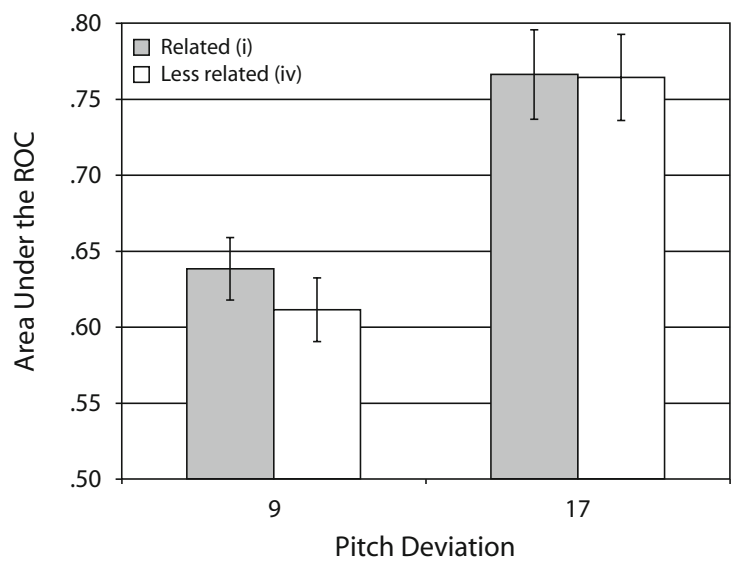

Figure 7. Areas under the receiver-operating characteristics (ROCs) for Experiment 3 presented as a function of tonal relatedness (related tonic, $i$; less related subdominant, iv) and pitch discrimination $(0 / 9,0 / 17$ cents). Chance level is at .5. Error bars represent standard errors.

$(F<1)$. The main effect of pitch discrimination was significant $\left[F(1,41)=90.70, M S_{\mathrm{e}}=91.22, p<.0001\right]$, with better performance when targets were lowered in pitch by 17 cents than when lowered by 9 cents. The main effect of tonal relatedness was not significant $(p=.14)$. For the 0/9-cent discrimination, Cohen's $d$ was calculated to measure the size of the tonal relatedness effect. The effect size was $d=.35$, which is categorized by J. Cohen (1988) as a small effect (small effects: $.20<d<.50$ ).

Hits and false alarms were analyzed by a $2 \times 3$ ANOVA with tonal relatedness (tonic or subdominant) and pitch deviation (in tune, 9 cents, or 17 cents) as withinparticipants factors. Only the effect of pitch deviation was significant $\left[F(2,82)=70.12, M S_{\mathrm{e}}=.065, p<.001\right.$; see Table 2]. Hits and false alarms for 17-cent targets were slightly more numerous for tonics than for subdominants, whereas false alarms for 9-cent targets were slightly more numerous for subdominants than for tonics, but none of these differences was significant. ${ }^{3}$

\section{Discussion}

Experiment 3 studied the influence of tonal relatedness on pitch perception with a comparison judgment task, using the sensitivity of a rating scale paradigm. Since the task was a same/different comparison of two tones, no internal reference pitch was needed, and a possibly

Table 2

Hits and False Alarms (FAs) in Experiment 3 Presented As a Function of Tonal Relatedness and Pitch Deviation (With Standard Errors)

\begin{tabular}{lcccc}
\hline & \multicolumn{4}{c}{ Tonal Relatedness } \\
\cline { 2 - 3 } Pitch Deviation & $M$ & $S E$ & & \multicolumn{2}{c}{ Less Related (iv) } \\
\cline { 2 - 5 } \cline { 5 - 6 } & $M$ & 3.59 & 63.7 & $S .49$ \\
\hline Hits & 64.9 & 1.92 & 42.8 & 2.22 \\
FA, 9 cents & 42.6 & 2.60 & 17.3 & 2.35 \\
FA, 17 cents & 18.5 & &
\end{tabular}


confounding influence of tonal relatedness on mistuning/ wrongness judgments should also have been avoided. ${ }^{4}$ Furthermore, the same/different task allowed us to test discrimination for small mistunings. A 9-cent mistuning $(0.5 \%)$ is close to the discrimination threshold for nonmusicians $(0.86 \%$, according to Micheyl, Delhommeau, Perrot, \& Oxenham, 2006).

For the 9-cent mistuning, area scores revealed an influence of the tonal relatedness of the to-be-compared tones: Pitch discrimination was better when the target was tonally related than when it was less related. This result strengthens the results of Experiments 1 and 2 showing that tonal expectations influence pitch perception. Although the observed effect size of tonal relatedness on pitch discrimination was in the small effects range (J. Cohen's, 1988, classification), the psychological relevance of an effect also depends on how minimal a manipulation of the independent variable can be and still produce the effect (Prentice \& Miller, 1992). In our study, the change in tonal relatedness was rather subtle (tonic vs. subdominant, as opposed to, e.g., tonic vs. leading tone) and was instantiated by a one-note difference in related and less related contexts.

The influence of tonal relatedness on pitch discrimination was observed only for the finer mistuning, but not for the 17-cent mistuning. This finding suggests that the influence of tonal expectations on pitch discrimination may come to influence performance only when the to-be-made comparisons are sufficiently fine-grained. Even if performance level is not at ceiling for the 17-cent mistuning, the required comparison may be too coarse, and thus the task may be too easy, so that facilitated processing based on tonal expectations has no apparent effect.

It is worth emphasizing that the participants could have made the same/different judgments while ignoring the preceding context, since the beginning melodic context was irrelevant for the task. This strategy would have been particularly possible because no internal reference pitch was needed (in contrast to the tasks in Experiments 1 and 2). Nevertheless, fine pitch judgments were influenced by the tonal function of the target tones. This result indicates that listeners process the tonal hierarchy of the context and automatically develop tonal expectations. Automatic musical expectations have been previously shown for chord processing with the harmonic-priming paradigm (Bigand, Tillmann, Poulin, D’Adamo, \& Madurell, 2001; Justus \& Bharucha, 2001; Tillmann \& Bigand, 2004; Tillmann, Janata, Birk, \& Bharucha, 2003).

\section{GENERAL DISCUSSION}

Our study showed the influence of tonal expectations on pitch perception with three experimental tasks: The pitch of related target tones was more accurately judged (i.e., via tuning/mistuning judgments) than the pitch of less related target tones, even if related targets were judged more in tune overall (Experiment 1); a correctly tuned target tone was processed more quickly when tonally related to the context than when less related (Experiment 2); and discrimination sensitivity for slightly mistuned tones was improved when these tones were tonally related to the context (Experiment 3).

The influence of Western tonal context on pitch perception had been shown for the detection of mistunings (Francès, 1958/1988; Lynch \& Eilers, 1992) and subjective pitch judgments (Warrier \& Zatorre, 2002). This previous research contrasted Western tonal contexts to non-Western contexts (e.g., Lynch \& Eilers, 1992) or to single-tone, repeated tone, and random contexts (Warrier et al., 1999; Warrier \& Zatorre, 2002). Our study investigated the influence of tonal context on a more fine-grained level within a tonality: Differences in pitch perception were observed between tones inside a tonal context-notably, by comparing target tones close in tonal relatedness. Through the use of pairs of almost identical tonal melodies, our data suggest that knowledge-based expectations can elicit different degrees of facilitation while remaining inside a tonal context. The findings indicate the strength of tonal knowledge in nonmusician listeners (i.e., listeners without explicit musical background) and stress the importance of implicit knowledge in auditory perception - notably, via the influence of tonal expectations on the processing of low-level sensory features such as pitch.

Psychoacoustic research on contextual pitch perception has shown improved pitch processing due to direct cues (Greenberg \& Larkin, 1968; Scharf, 1998) and indirect cues (Hafter et al., 1993; Howard et al., 1984). Expectations based on bottom-up patterns (notably, following Gestalt properties) also exist in music perception (Narmour, 1990; Schellenberg et al., 2002). Their influence on pitch processing has been investigated by Jones, Johnston, and Puente (2006): Contextual patterns of pitch intervals influence the detection of a change in pitch height of the pattern's penultimate tone. In our study, these pattern-like influences (e.g., linked to pitch proximity or continuity) have been kept constant between the melodies of a pair in order to focus on the influence of listeners' tonal knowledge. Our study thus took the investigation of central factors in pitch processing one step further by showing an effect of tonal knowledge - that is, a top-down effect of a set of abstract features that are not contingent on the experimental session and the immediately preceding tone pattern.

Some top-down influences on pitch processing have been reported with a cued two-alternative forced choice task (Green \& McKeown, 2001, Experiment 3). The percentages of valid cues (i.e., cues that had the same frequency as the signal) were either $75 \%$ or $25 \%$ (i.e., informative and uninformative, respectively, about the frequency of the signal). The observed cuing effect was larger when the cues were informative. The authors suggested that listeners were intentionally trying to focus on the frequency of the cue when it was likely to predict the signal frequency (resulting in a more accurate pitch processing). These topdown processes were contingent upon the experimental session and were deliberate. On the contrary, top-down processes in our study refer to tonal knowledge, which results from a long exposure to the statistical regularities of Western music stored in long-term memory.

Another difference from Green and McKeown's (2001) study is that in our study, the listeners' tonal knowledge did 
not provide direct information about the task and could not serve for the development of task-related strategies. The fact that tonal relatedness was irrelevant to the tasks pleads for the automaticity of the induced top-down processes. This is in agreement with harmonic-priming data showing that chord processing is influenced automatically by schematic expectations despite conflicting veridical expectations (Justus \& Bharucha, 2001; Tillmann \& Bigand, 2004).

In psychoacoustic studies, contextual pitch perception effects have been explained in terms of attentional bands or filters, which are formed around the cued frequency: A cue draws listeners' attention to a narrow frequency band centered on the cue frequency, and detection performance is best at the cue frequency and progressively decreases with increased frequency distance between the cue and the target (Greenberg \& Larkin, 1968; Scharf, 1998; Scharf, Quigley, Aoki, Peachey, \& Reeves, 1987). Attentional bands can be multiple in case of multiple cues (e.g., Scharf, 1998) and can be based on specific relations (e.g., musical fifths; Hafter et al., 1993) to a cue. Although our study differs in many aspects from these psychoacoustic studies, the concept of attentional filters may be applied here: Tonal knowledge may lead to an attentional band centered on the tonic pitch, providing improved processing accuracy for the tonic pitch (in comparison with the subdominant pitch). In our experimental materials, the pitch of the targets differed between melodies, and the pitch of the tonic depended on the key instilled by the melodic context. The observed influence of tonal relatedness on pitch processing thus suggests that attentional bands can change from trial to trial on the basis of the contextual tonal center. Consistent with this potential framework, the relevance of attentional bands for music perception has been previously discussed by Bharucha (1996) for the phenomenon of melodic anchoring.

Our data showed top-down influences on pitch processing in musical contexts. Since performance in behavioral tasks reflects the outcome of several processing stages, the question remains whether the performance benefit is a direct consequence of knowledge-based processes on low-level, perceptual processes, whether it is mediated by attentional processes (which then influence perceptual processes), whether it is a consequence of decisional or response-related processes, or whether it is a mixture of these processes. This question is not specific to music perception but applies also to visual and auditory perception. For speech perception, the question is whether the influence of lexical knowledge is restricted to postperceptual decision processes or extends to prelexical perceptual processes (see McClelland, Mirman, \& Holt, 2006; McQueen, Norris, \& Cutler, 2006; Mirman, McClelland, \& Holt, 2006). Using a behavioral approach, Samuel (2001), for example, provided evidence for the influence of topdown lexical processes on the perceptual phenomenon of selective adaptation based on repeated sound processing. To separate perceptual sensitivity from response bias, SDT was applied to the analyses of discrimination performance. Data from sensitivity measures have been taken as evidence for the influence of attention on early perceptual processes in visual perception (Correa, Lupiáñez, \&
Tudela, 2005; Hawkins et al., 1990; Luck et al., 1994). For example, temporal expectations increased sensitivity in a perceptual discrimination task without changing the response criterion (Correa et al., 2005). In our study, the SDT analyses using area scores (Experiment 1 and, particularly, Experiment 3) indicated top-down influences (here, based on tonal knowledge) on bias-free discrimination performance and thus suggested that there were influences on perceptual levels.

Although it is difficult to provide evidence for specific processing levels by means of behavioral methods, measuring neurophysiological responses to expected and unexpected stimuli can help to unveil the levels at which top-down processes modulate event processing. Research on visual and auditory perception has shown that early electrophysiological markers reflecting perceptual processing can be influenced by top-down processes (e.g., Correa, Lupiáñez, Madrid, \& Tudela, 2006). In the visual modality, spatial orienting of attention influenced not only sensitivity in detection performance, but also the amplitude of the N1-P1 complex in the event-related potential (ERP) data (Luck et al., 1994). Similarly, temporal attention can influence early visual processing, as reflected in the modulation of N1 components (Correa et al., 2006). For auditory perception, early processing stages (i.e., N1 components) have been modulated by spatial, as well as temporal, attention (e.g., Hillyard, Hink, Schwent, \& Picton, 1973; Lange, Krämer, \& Röder, 2006; Lange, Rösler, $\&$ Röder, 2003). Selective attention can influence auditory processing not only at the level of the auditory cortex (including the sharpness of the tuning curves; Kauramäki, Jääskeläinen, \& Sams, 2007), but also at the levels of the brain stem and, probably, the cochlea (Giard, Fort, Mouchetant-Rostaing, \& Pernier, 2000).

This brief review of neurophysiological studies shows that top-down influences on perceptual processes have been studied mostly in terms of the effects of attentional processes and expectations on the processing of temporal and spatial information. For music perception, links between musical structures and attention have been proposed in a dynamic theory of attending (Jones, 1987; Jones \& Boltz, 1989). In this theory, musical events that are important in the melodic and temporal structure influence the modulation of attention over time, directing attentional resources to the processing of structurally important events. In the priming paradigm, more attentional resources would be allocated to tonally related than to less related targets, resulting in facilitation effects (see Bigand et al., 2001; Escoffier \& Tillmann, 2008). The influence of tonally induced top-down expectations could thus modulate perceptual processes, such as pitch processing, via attention. Our study provided behavioral data suggesting that top-down, knowledge-based processes modulate pitch processing in music perception. Electrophysiological evidence for differences in N1 amplitude influenced by tonal relatedness has been recently reported for tones in music-like contexts, even within the constraints of an MMN paradigm, albeit in an active-listening situation (Krohn, Brattico, Välimäki, \& Tervaniemi, 2006). Using ERPs with materials controlled for sensory expectations, like the melodies used here, will 
allow us to further investigate the perceptual processes influenced by cognitive expectations - expectations linked to listeners' tonal knowledge.

\section{AUTHOR NOTE}

This research was supported by a "Junior Research Team" grant from the French Ministry of Research. Correspondence concerning this article should be addressed to F. Marmel, Neurosciences Sensorielles Comportement Cognition, Université Claude Bernard-Lyon I, CNRS UMR 5020, 50 Av. Tony Garnier, F-69366 Lyon Cedex 07, France (e-mail: frederic.marmel@olfac.univ-lyon1.fr).

\section{REFERENCES}

Bharucha, J. J. (1984). Event hierarchies, tonal hierarchies, and assimilation: A reply to Deutsch and Dowling. Journal of Experimental Psychology: General, 113, 421-425.

Bharucha, J. J. (1996). Melodic anchoring. Music Perception, 13, 383-400.

Bharucha, J. J., \& Stoeckig, K. (1986). Reaction time and musical expectancy: Priming of chords. Journal of Experimental Psychology: Human Perception \& Performance, 12, 403-410.

Bharucha, J. J., \& Stoeckig, K. (1987). Priming of chords: Spreading activation or overlapping frequency spectra? Perception \& Psychophysics, 41, 519-524.

BIGAND, E. (1997). Perceiving musical stability: The effect of tonal structure, rhythm, and musical expertise. Journal of Experimental Psychology: Human Perception \& Performance, 23, 808-822.

Bigand, E., Madurell, F., Tillmann, B., \& Pineau, M. (1999). Effect of global structure and temporal organization on chord processing Journal of Experimental Psychology: Human Perception \& Performance, 25, 184-197.

Bigand, E., \& Pineau, M. (1997). Global context effects on musical expectancy. Perception \& Psychophysics, 59, 1098-1107.

Bigand, E., Poulin, B., Tillmann, B., Madurell, F., \& D’Adamo, D. A. (2003). Sensory versus cognitive components in harmonic priming. Journal of Experimental Psychology: Human Perception \& Performance, 29, 159-171.

Bigand, E., \& Poulin-Charronnat, B. (2006). Are we "experienced listeners"? A review of the musical capacities that do not depend on formal musical training. Cognition, 100, 100-130.

Bigand, E., Tillmann, B., Poulin, B., D'Adamo, D. A., \& MaDURELL, F. (2001). The effect of harmonic context on phoneme monitoring in vocal music. Cognition, 81, B11-B20.

Bigand, E., Tillmann, B., Poulin-Charronnat, B., \& ManderLIER, D. (2005). Repetition priming: Is music special? Quarterly Journal of Experimental Psychology, 58A, 1347-1375.

Brattico, E., NÄ̈̈täNen, R., \& TervaniEmi, M. (2001). Context effects on pitch perception in musicians and nonmusicians: Evidence from event-related-potential recordings. Music Perception, 19, 199-222.

CoHEN, J. (1988). Statistical power analysis for the behavioral sciences (2nd ed.). Hillsdale, NJ: Erlbaum.

Cohen, J. [D.], MacWhinney, B., Flatt, M., \& Provost, J. (1993). PsyScope: An interactive graphic system for designing and controlling experiments in the psychology laboratory using Macintosh computers. Behavior Research Methods, Instruments, \& Computers, 25 , 257-271. [For current OS X version, see psy.ck.sissa.it]

Correa, Á., Lupiáñez, J., Madrid, E., \& Tudela, P. (2006). Temporal attention enhances early visual processing: A review and new evidence from event-related potentials. Brain Research, 1076, 116-128.

Correa, Á., Lupiáñez, J., \& Tudela, P. (2005). Attentional preparation based on temporal expectancy modulates processing at the perceptual level. Psychonomic Bulletin \& Review, 12, 328-334.

Dowling, W. J., KWAK, S., \& AndREws, M. W. (1995). The time course of recognition of novel melodies. Perception \& Psychophysics, 57, 136-149.

Dowling, W. J., Tillmann, B., \& Ayers, D. F. (2001). Memory and the experience of hearing music. Music Perception, 19, 249-276.

Escoffier, N., \& Tillmann, B. (2008). The tonal function of a taskirrelevant chord modulates speed of visual processing. Cognition, 107, 1070-1083.
Francès, R. (1988). The perception of music (W. J. Dowling, Trans.). Hillsdale, NJ: Erlbaum. (Original work published 1958)

Frankland, B., \& Cohen, A. J. (1990). Expectancy profiles generated by major scales: Group differences in ratings and reaction time. Psychomusicology, 9, 173-192.

Geringer, J. M., \& Madsen, C. K. (1984). Pitch and tempo discrimination in recorded orchestral music among musicians and nonmusicians. Journal of Research in Music Education, 32, 195-204.

Giard, M.-H., Fort, A., Mouchetant-Rostaing, Y., \& Pernier, J. (2000). Neurophysiological mechanisms of auditory selective attention in humans. Frontiers in Bioscience, 5, D84-D94.

Gottselig, J. M., Brandeis, D., Hofer-Tinguely, G., Borbély, A. A., \& ACHERmanN, P. (2004). Human central auditory plasticity associated with tone sequence learning. Learning \& Memory, 11, 162-171.

Green, T. J., \& McKeown, J. D. (2001). Capture of attention in selective frequency listening. Journal of Experimental Psychology: Human Perception \& Performance, 27, 1197-1210.

GreenBerG, G. Z., \& LARKIN, W. D. (1968). Frequency-response characteristic of auditory observers detecting signals of a single frequency in noise: The probe-signal method. Journal of the Acoustical Society of America, 44, 1513-1523.

Hafter, E. R., Schlauch, R. S., \& TANG, J. (1993). Attending to auditory filters that were not stimulated directly. Journal of the Acoustical Society of America, 94, 743-747.

Hawkins, H. L., Hillyard, S. A., Luck, S. J., Mouloua, M., DownING, C. J., \& WoodWARD, D. P. (1990). Visual attention modulates signal detectability. Journal of Experimental Psychology: Human Perception \& Performance, 16, 802-811.

Hébert, S., Peretz, I., \& Gagnon, L. (1995). Perceiving the tonal ending of tune excerpts: The roles of pre-existing representation and musical expertise. Canadian Journal of Experimental Psychology, 49, 193-209.

Hillyard, S. A., Hink, R. F., Schwent, V. L., \& Picton, T. W. (1973). Electrical signs of selective attention in the human brain. Science, 182, 177-180.

Howard, J. H., Jr., O'Toole, A. J., Parasuraman, R., \& Bennett, K. B. (1984). Pattern-directed attention in uncertain-frequency detection. Perception \& Psychophysics, 35, 256-264.

Johnston, H. M., \& Jones, M. R. (2006). Higher order pattern structure influences auditory representational momentum. Journal of Experimental Psychology: Human Perception \& Performance, 32, 2-17.

Jones, M. R. (1987). Dynamic pattern structure in music: Recent theory and research. Perception \& Psychophysics, 41, 621-634.

Jones, M. R., \& Boltz, M. (1989). Dynamic attending and responses to time. Psychological Review, 96, 459-491.

Jones, M. R., Johnston, H. M., \& Puente, J. (2006). Effects of auditory pattern structure on anticipatory and reactive attending. Cognitive Psychology, 53, 59-96.

Justus, T. C., \& BhaRUCHA, J. J. (2001). Modularity in musical processing: The automaticity of harmonic priming. Journal of Experimental Psychology: Human Perception \& Performance, 27, 1000-1011.

KAURAmÄKI, J., JÄÄSKeläInEN, I. P., \& SAms, M. (2007). Selective attention increases both gain and feature selectivity of the human auditory cortex. PLOS ONE, 2, e909. doi:10.1371/journal.pone.0000909.

Krohn, K. I., Brattico, E., VÄlimäKi, V., \& Tervaniemi, M. (2006). Neural representations of the hierarchical scale pitch structure. Music Perception, 24, 281-296.

KrumhansL, C. L. (1979). The psychological representation of musical pitch in a tonal context. Cognitive Psychology, 11, 346-374.

KRUMHANSL, C. L. (1990). Cognitive foundations of musical pitch. New York: Oxford University Press.

LANGE, K., Krämer, U. M., \& RöDER, B. (2006). Attending points in time and space. Experimental Brain Research, 173, 130-140.

LANGE, K., Rösler, F., \& RöDER, B. (2003). Early processing stages are modulated when auditory stimuli are presented at an attended moment in time: An event-related potential study. Psychophysiology, 40, 806-817.

Luck, S. J., Hillyard, S. A., Mouloua, M., WoldorfF, M. G., Clark, V. P., \& Hawkins, H. L. (1994). Effects of spatial cuing on luminance detectability: Psychophysical and electrophysiological evidence for early selection. Journal of Experimental Psychology: Human Perception \& Performance, 20, 887-904. 
LYNCH, M. P., \& EILERS, R. E. (1992). A study of perceptual development for musical tuning. Perception \& Psychophysics, 52, 599-608.

McClelland, J. L., Mirman, D., \& Holt, L. L. (2006). Are there interactive processes in speech perception? Trends in Cognitive Sciences, 10, 363-369.

McQueen, J. M., Norris, D., \& Cutler, A. (2006). Are there really interactive processes in speech perception? Trends in Cognitive Sciences, 10, 533.

Micheyl, C., Delhommeau, K., Perrot, X., \& Oxenham, A. J. (2006). Influence of musical and psychoacoustical training on pitch discrimination. Hearing Research, 219, 36-47.

Mirman, D., McClelland, J. L., \& Holt, L. L. (2006). Response to McQueen et al.: Theoretical and empirical arguments support interactive processing. Trends in Cognitive Sciences, 10, 534.

NARMour, E. (1990). The analysis and cognition of basic melodic structures: The implication-realization model. Chicago: University of Chicago Press.

Prentice, D. A., \& Miller, D. T. (1992). When small effects are impressive. Psychological Bulletin, 112, 160-164.

SAmUEL, A. G. (2001). Knowing a word affects the fundamental perception of the sounds within it. Psychological Science, 12, 348-351.

Scharf, B. (1998). Auditory attention: The psychoacoustical approach. In H. Pashler (Ed.), Attention (pp. 75-117). Hove, U.K.: Psychology Press.

Scharf, B., Quigley, S., Aoki, C., Peachey, N., \& Reeves, A. (1987) Focused auditory attention and frequency selectivity. Perception \& Psychophysics, 42, 215-223.

Schellenberg, E. G., Adachi, M., Purdy, K. T., \& McKinnon, M. C. (2002). Expectancy in melody: Tests of children and adults. Journal of Experimental Psychology: General, 131, 511-537.

SwETs, J. A. (1973). The relative operating characteristic in psychology. Science, 182, 990-1000.

TAnner, W., \& Norman, R. (1954). The human use of information: II. Signal detection for the case of an unknown signal parameter. Transactions of the Institute of Radio Engineering, Professional Group on Information Theory, 4, 222-227.

Tekman, H. G., \& Bharucha, J. J. (1992). Time course of chord priming. Perception \& Psychophysics, 51, 33-39.

Tillmann, B., Bharucha, J. J., \& Bigand, E. (2000). Implicit learning of tonality: A self-organizing approach. Psychological Review, 107, 885-913.

Tillmann, B., \& Bigand, E. (2004). Musical priming: Schematic expectations resist repetition priming. In S. D. Lipscomb, R. Ashley, R. O. Gjerdingen, \& P. Webster (Eds.), Proceedings of the 8th International Conference on Music Perception and Cognition (pp. 674-676). Adelaide, Australia: Causal Productions.

Tillmann, B., Bigand, E., Escoffier, N., \& Lalitte, P. (2006). The influence of musical relatedness on timbre discrimination. European Journal of Cognitive Psychology, 18, 343-358.

Tillmann, B., Bigand, E., \& Pineau, M. (1998). Effects of global and local contexts on harmonic expectancy. Music Perception, 16, 99-118.

Tillmann, B., Janata, P., Birk, J., \& Bharucha, J. J. (2003). The costs and benefits of tonal centers for chord processing. Journal of
Experimental Psychology: Human Perception \& Performance, 29, 470-482.

Verde, M. F., Macmillan, N. A., \& Rotello, C. M. (2006). Measures of sensitivity based on a single hit rate and false alarm rate: The accuracy, precision, and robustness of $d^{\prime}, A_{z}$, and $A^{\prime}$. Perception \& Psychophysics, 68, 643-654.

Warrier, C. M., Belin, P., Merlet, I., \& Zatorre, R. J. (1999). fMrI study examining effect of melodic context on pitch discrimination. Society for Neuroscience Abstracts, 25, 1629.

WARrier, C. M., \& ZATORre, R. J. (2002). Influence of tonal context and timbral variation on perception of pitch. Perception \& Psychophysics, 64, 198-207.

Watson, C. S., \& Foyle, D. C. (1985). Central factors in the discrimination and identification of complex sounds. Journal of the Acoustical Society of America, 78, 375-380.

Watson, C. S., Kelly, W. J., \& Wroton, H. W. (1976). Factors in the discrimination of tonal patterns: II. Selective attention and learning under various levels of stimulus uncertainty. Journal of the Acoustical Society of America, 60, 1176-1186.

\section{NOTES}

1. Our study focused, like previous music perception studies (Bigand \& Pineau, 1997; Bigand, Poulin, Tillmann, Madurell, \& D’Adamo, 2003; Bigand, Tillmann, Poulin, D’Adamo, \& Madurell, 2001; Tillmann, Janata, Birk, \& Bharucha, 2003), on cognitive expectations based on the tonal hierarchy - that is, the schematic knowledge of tonal relations that is independent of a specific musical piece. The tonal hierarchy has to be distinguished from the event hierarchy (Bharucha, 1984), the hierarchy of events for a specific musical piece that combines the abstract tonal hierarchy with the specific chaining of musical events in time (their order and temporal positions).

2. A first version of this paradigm using a -17 -cent mistuning was too difficult: Four out of 8 participants (instrumental instruction from 0 to 16 years, with a mean of 6.3 years $[S D=5.5]$ and a median of 5.5) had correct responses inferior to $55 \%$.

3. An analysis of median ratings revealed a similar pattern of results: A marginally significant interaction between tonal relatedness and pitch deviation was observed $\left[F(2,82)=3.00, M S_{\mathrm{e}}=.011, p=.055\right]$, with weaker ratings for tonics than for subdominants with 9-cent targets. This result suggests that 9-cent targets were perceived as more out of tune when they functioned as tonics than when they functioned as subdominants.

4. In contrast to Experiment 1, no significant response bias was observed, even if hits and false alarms were slightly more numerous for 17-cent targets in the related than in the less-related condition. This difference between the experiments may have been due to the samel different task (avoiding a possible confound between mistuning and tonal function) and to the error feedback, which may have imposed a fixed criterion, thus preventing a bias shift.

(Manuscript received June 4, 2007; revision accepted for publication February 1, 2008.) 